\title{
20 PATIENT AND PROVIDER PERCEPTIONS OF ADVANCE CARE PLANNING USING ELECTRONIC HEALTH RECORD PATIENT PORTALS
}

S Bose-Brill', C Kobe, T Pressler, Celia Wills ${ }^{1}$ Ohio State University

10.1136/bmjspcare-2012-000250.20

The electronic health record (EHR) and EHR-tethered patient portals provide innovative opportunities for patient-provider advance care planning (ACP) communication. Development of patient-centred ACP tools for EHR-tethered patient portals requires investigation of barriers to use. An ongoing survey study in Ohio State University primary care clinics is assessing willingness to use OSUMyChart (an EHR-tethered patient portal) for ACP communication. This is phase one of a multiphase project developing a patient-provider ACP communication framework. 27 of 30 physician respondents $(90 \%)$ indicate that EHR could improve ACP delivery. 34 of 39 patient respondents $(87.2 \%)$ would respond positively to a provider-initiated ACP discussion. However, only 20 patients (51.2\%) are willing to use EHR to discuss ACP. Patients responding negatively to ACP EHR communication have lower rates of internet access ( $81 \%$ vs. $44 \%$ ) and OSUMyChart activation ( $52 \%$ vs. $17 \%$ ) than patients who respond positively. Most unwilling patients without internet cite lack of access as the sole reason for reluctance to EHR-based ACP. Possible solutions are on-site technical support and partnerships subsidising internet connectivity for patients without access.

DevelopEH EHR-tethered patient portals for the purposes of facilitating ACP must be coupled with strategies for combating health disparities arising from limitations in electronic connectivity. This presentation will describe: (1) patient and provider responses to potential use of EHR-tethered patient portals in ACP; (2) characteristics of patients conveying reluctance to use electronic resources for ACP communication; (3) strategies for overcoming barriers to EHR-based ACP communication. 